\title{
Prevalence, risk factors and microorganisms of urinary tract infections in patients with type 2 diabetes mellitus: a retrospective study in China
}

This article was published in the following Dove Press journal:

Therapeutics and Clinical Risk Management

$\mathrm{Ke} \mathrm{He}^{1,2, *}$

Yun $\mathrm{Hu}^{2, *}$

Jun-Cheng Shi

Yun-Qing Zhu²

Xiao-Ming Mao ${ }^{2}$

'Department of Endocrinology,

Wuxi Hospital of Traditional Chinese Medicine, Wuxi Hospital Affiliated to Nanjing University of Chinese Medicine, Wuxi, China; ${ }^{2}$ Department of Endocrinology, Nanjing First Hospital, Nanjing Medical University, Nanjing, China

*These authors contributed equally to this work
Correspondence: Xiao-Ming Mao Department of Endocrinology, Nanjing First Hospital, Nanjing Medical University, 68 ChangLe St, Nanjing 210006, China Tel +86 I50 6|82 222। Email stefanie8807@I26.com
Background: Urinary tract infections (UTIs) occur more frequently in diabetic patients. This study was conducted to investigate the prevalence, risk factors and microorganisms of UTIs in Chinese patients with type 2 diabetes (T2D).

Patients and methods: A total of 3,652 Chinese inpatients with T2D were reviewed and data on their clinical characteristics, symptoms of UTIs, random blood glucose, HbA1c, glutamic acid decarboxylase antibody, insulin autoantibody, albumin excretion rate in 24-hour urine, urine culture and susceptibility to antibiotics, and so on were collected. Binary logistic analysis was performed to look for risk factors of UTIs.

Results: There were 409 (11.2\%) patients suffering from UTIs. Gender, age, random blood glucose, insulin autoantibody and albumin excretion rate in 24-hour urine were the risk factors of UTIs in diabetic patients. The percentage of positive urine cultures was higher in the asymptomatic bacteriuria patients than in symptomatic patients $(P<0.001)$. The incidence of septicemia was considerable in the UTIs and asymptomatic bacteriuria groups. Escherichia coli was the most common pathogenic microorganism isolated in diabetic patients with UTIs, and one-half of the E. coli infections were multidrug resistant. Furthermore, meropenem was the most effective antibiotic on E. coli.

Conclusion: We suggest that a routine urine analysis or urine culture should be conducted in patients with T2D diabetes who have the identified risk factors. The UTIs might affect the islet function or blood glucose control in patients with T2D. Before a doctor decides to prescribe antibiotics to a diabetic patient with UTIs, the drug sensitivity test should be performed.

Keywords: urinary tract infections, asymptomatic bacteriuria, diabetes, multidrug resistant

\section{Introduction}

Patients with diabetes are susceptible to infections (about four times more than non-diabetics), ${ }^{1,2}$ which might be ascribed to their abnormalities in immune function. ${ }^{3,4}$ Among the infections, urinary tract infections (UTIs) occur more frequently in diabetic patients ${ }^{5,6}$ because of urine glucose excretion and chronic neurologic bladder dysfunction. $^{7}$ Furthermore, the prevalence of asymptomatic bacteriuria (ASB) in diabetic patients is three times higher than in normal people; however, whether the symptomatic UTIs are preceded by ASB is unknown.,

UTIs may cause serious complications in diabetic patients, such as emphysematous cystitis, renal failure, bacteremia and papillary necrosis. ${ }^{10,11}$ UTIs can also cause systemic inflammation and oxidative stress that elevate blood glucose and increase insulin resistance. Although the incidence of UTIs is higher and the severity of UTIs is more than anyone thought of previously, less than half of the UTI patients seek treatment in Asia. ${ }^{12}$ 
Escherichia coli is the frequent uropathogen in UTIs. However, the incidences of UTIs in different races and ethnicities are different. In previous studies, it was reported that the isolation of $E$. coli in European patients with UTIs was decreasing in the past 15 years. ${ }^{13,14}$ The isolation rate of $E$. coli was $26 \%$ in Japanese patients with UTIs and $55.1 \%$ in Indian patients. Both these studies were published in 2014..$^{15,16}$

In this study, we reviewed 3,653 Chinese patients with type 2 diabetes (T2D) and studied the prevalence and characteristics of UTIs. The objective of this study is to find the risk factors and responsible pathogenetic microorganisms of UTIs in Chinese patients with T2D.

\section{Patients and methods}

\section{Patient selection}

The study protocol and patient consent forms were approved by the Institutional Ethical Committee of Nanjing First Hospital, Nanjing Medical University. All patients gave written informed consent to participate in the study. All procedures performed in studies involving human participants were in accordance with the ethical standards of the institutional and national research committee and with the 1964 Helsinki Declaration and its later amendments or comparable ethical standards. The methods were carried out in accordance with the Declaration of Helsinki guidelines, including any relevant details.

A total of 3,652 Chinese patients with T2D, who were hospitalized in the Department of Endocrinology, Nanjing First Hospital, Nanjing Medical University between March 2013 and July 2016, were included in this retrospective study. All of the patients were diagnosed with diabetes mellitus according to the American Diabetes Association diabetic definition standard by the admitting physician in our department before hospitalization. The exclusion criteria for patient selection were pregnancy and use of antimicrobial drugs in the last 14 days.

Patients were diagnosed as having UTIs if they met one of the following criteria or both: 1) pyuria (a leukocyte count of $>10 / \mathrm{mm}^{3}$ in clean-voided midstream urine specimen) in routine urine analysis of two urine samples, accompanied by symptoms of UTIs (including dysuria, pollakiuria, urgency, odynuria and fever) or not and 2) two positive midstream urine cultures (presence of at least $10^{5}$ colony forming units $/ \mathrm{mL}$ of Gram-negative bacteria or $10^{4}$ colony forming units $/ \mathrm{mL}$ of Gram-positive bacteria) with the same microorganism. Four hundred and nine of the patients were diagnosed with UTIs according to the criteria, and 388 patients who had other infections (such as respiratory tract infections, skin and mucus membrane infections, digestive tract infections) were excluded.

\section{Data collection}

The patients' age, gender, height, weight, presence or absence of symptoms of UTI, and the type, duration and treatment of diabetes were recorded. Measurements of random blood glucose (RBG; measured when they began hospitalization), HbA1c, fasting and 2-hour post-meal serum c-peptide, glutamic acid decarboxylase antibody (GAD-Ab), insulin autoantibody (IAA), total cholesterol, high-density lipoprotein cholesterol, triglycerides, low-density lipoprotein cholesterol, albumin excretion rate in 24-hour urine (UAlb), blood leukocyte count (total leukocytes and percentage of band forms), blood creatine ( $\mathrm{Cr}$ ), glutamate-pyruvate transaminase, potassium concentration, blood albumin and uric acid (measured during the first 3 days of hospitalization) were collected. The initial body mass index (BMI) of the patients was calculated as weight in kilograms divided by height in meters squared. Out of the total studied population, 345 patients underwent positive urine cultures and tests for susceptibility to antibiotics, and the results were recorded. An isolate was considered as multidrug resistant (MDR) if found resistant to three or more antimicrobials belonging to different classes/groups of antimicrobials.

\section{Statistical analysis}

Analyses were performed using the SPSS 16.0 (SPSS Inc., Chicago, IL, USA) statistical package. All variables were tested for normal distribution of the data. Data are presented as means \pm standard error or percentages. Differences between UTIs and non-UTIs were examined using the Student's unpaired $t$-test for parametric data or the MannWhitney $U$ test for nonparametric data. The categorical data were examined with chi-square test. Binary logistic analysis was performed to look for the risk factors of UTIs in the sample population.

\section{Results}

\section{Characteristics of the UTI patients}

Of the 3,652 patients with T2D in the study, aged between 18 and 94 years (mean age, 59.32 \pm 14.13 ), 409 patients were diagnosed as having UTIs. Among the 409 (11.2\%) UTI patients, 221 patients had no UTI symptoms and 9 patients had urethral catheters. Eleven patients had bloodstream infections accompanying UTIs and among them, six had 
no UTI symptoms. One of the patients died of serious infections. The incidence of UTIs was higher in female than in males $(24.13 \%$ vs $3.67 \% ; P<0.0001)$. Patients with UTIs were older and had longer duration of diabetes mellitus than non-UTI patients $(P<0.0001)$. Blood leukocyte count, $\mathrm{Cr}$ and UAlb increased in UTI patients, while uric acid, glutamate-pyruvate transaminase and blood albumin decreased (all with $P<0.005$ ). There were no differences in BMI and serum potassium between the two groups (Table 1). $\mathrm{RBG}, \mathrm{GAD}-\mathrm{Ab}$ and IAA increased in patients with UTIs $(P<0.001)$, while HbA1c, blood lipids and C-peptide were similar in patients with UTIs to those of non-UTI patients $(P>0.05$; Table 1$)$.

\section{Risk factors of UTIs}

Risk factors for UTIs were examined in stepwise binary logistic regression model. All factors associated with UTIs at $P<0.5$ in unadjusted analyses were included in the backward

Table I Characterization of patients with T2D

\begin{tabular}{|c|c|c|c|}
\hline Characteristics & Non-UTIs & UTIs & $P$-value \\
\hline Gender (male/female) & $(I, 783 / I, 072)$ & $(68 / 34 I)$ & $<0.001$ \\
\hline Age (years) & $57.43 \pm 0.26$ & $64.28 \pm 0.64$ & $<0.001$ \\
\hline Duration of DM (years) & $6.09 \pm 0.12$ & $7.66 \pm 0.36$ & $<0.001$ \\
\hline BMI $\left(\mathrm{kg} / \mathrm{m}^{2}\right)$ & $24.46 \pm 0.07$ & $24.33 \pm 0.18$ & 0.257 \\
\hline WBC $\left(\times 10^{9} / \mathrm{L}\right)$ & $6.35 \pm 0.06$ & $7.28 \pm 0.20$ & 0.001 \\
\hline $\mathrm{N}(\%)$ & $59.85 \pm 0.33$ & $64.67 \pm 0.65$ & $<0.001$ \\
\hline L (\%) & $32.5 I \pm 0.3 I$ & $28.65 \pm 0.59$ & $<0.001$ \\
\hline $\mathrm{Cr}(\mu \mathrm{mol} / \mathrm{L})$ & $74.66 \pm 1.56$ & $81.89 \pm 2.46$ & $<0.001$ \\
\hline $\mathrm{UA}(\mu \mathrm{mol} / \mathrm{L})$ & $332.46 \pm 4.04$ & $320.55 \pm 7.64$ & $<0.001$ \\
\hline $\mathrm{ALT}(\mathrm{U} / \mathrm{L})$ & $31.39 \pm 0.94$ & $27.39 \pm 1.4 \mid$ & $<0.001$ \\
\hline Alb $(g / L)$ & $4 I .5 I \pm 0.15$ & $38.82 \pm 0.26$ & $<0.001$ \\
\hline Blood $\mathrm{K}^{+}(\mathrm{mmol} / \mathrm{L})$ & $3.93 \pm 0.01$ & $3.91 \pm 0.03$ & 0.279 \\
\hline UAlb (mg/L) & $66.40 \pm 3.48$ & $104.85 \pm 14.66$ & 0.001 \\
\hline RBG (mmol/L) & $11.7 I \pm 0.14$ & $13.03 \pm 0.40$ & 0.003 \\
\hline $\mathrm{HbAlc}(\%)$ & $9.48 \pm 0.04$ & $9.54 \pm 0.13$ & 0.952 \\
\hline $\mathrm{FC}-\mathrm{P}(\mathrm{ng} / \mathrm{mL})$ & $\mathrm{I} .77 \pm 0.03$ & $1.86 \pm 0.06$ & 0.256 \\
\hline $2 \mathrm{hC}-\mathrm{P}(\mathrm{ng} / \mathrm{mL})$ & $4.0 \mathrm{I} \pm 0.06$ & $4.07 \pm 0.17$ & 0.792 \\
\hline GAD-Ab (IU/mL) & $20.99 \pm 0.57$ & $23.95 \pm 1.47$ & $<0.001$ \\
\hline IAA (\%) & $5.95 \pm 0.19$ & $7.4 I \pm 0.58$ & $<0.001$ \\
\hline $\mathrm{TG}(\mathrm{mmol} / \mathrm{L})$ & $2.10 \pm 0.04$ & $1.92 \pm 0.09$ & 0.105 \\
\hline $\mathrm{TC}(\mathrm{mmol} / \mathrm{L})$ & $5.10 \pm 0.03$ & $5.12 \pm 0.07$ & 0.957 \\
\hline HDL-c (mmol/L) & $\mathrm{I} .37 \pm 0.0 \mathrm{I}$ & $1.40 \pm 0.02$ & 0.063 \\
\hline LDL-c (mmol/L) & $2.95 \pm 0.12$ & $2.80 \pm 0.04$ & 0.617 \\
\hline
\end{tabular}

Abbreviations: 2hC-P, 2-hour post-meal serum c-peptide; Alb, blood albumin; $\mathrm{ALT}$, glutamate-pyruvate transaminase; BMI, body mass index; $\mathrm{Cr}$, blood creatine; DM, diabetes mellitus; FC-P, fasting serum c-peptide; GAD-Ab, glutamic acid decarboxylase antibody; HDL-c, high-density lipoprotein cholesterol; IAA, insulin autoantibody; L, lymphocyte; LDL-c, low-densitylipoprotein cholesterol; N, neutrophil; RBG, random blood glucose; T2D, type 2 diabetes; TC, total cholesterol; TG, triglycerides; UA, uric acid; UAlb, albumin excretion rate in 24-hour urine; UTI, urinary tract infection; WBC, white blood cell.
Table 2 Risk factors for UTIs in diabetic patients

\begin{tabular}{llll}
\hline Risk factors & OR & $\mathbf{9 5 \% ~ C l}$ & $P$-value \\
\hline Gender (female) & 10.665 & $7.016-16.211$ & $<0.001$ \\
Age & 1.019 & $1.006-1.032$ & 0.005 \\
RBG & 1.034 & $1.013-1.055$ & 0.001 \\
IAA & 1.015 & $1.002-1.028$ & 0.022 \\
UAlb & 1.001 & $1.001-1.002$ & $<0.001$ \\
\hline
\end{tabular}

Abbreviations: IAA, insulin autoantibody; OR, odds ratio; RBG, random blood glucose; UAlb, albumin excretion rate in 24-hour urine; UTI, urinary tract infection.

selection regression model and odds ratios and $95 \% \mathrm{CI}$ were derived. Gender, age, duration of diabetes, BMI, HbA1c, fasting and 2-hour post-meal serum c-peptide, RBG, IAA, GAD-Ab, high-density lipoprotein cholesterol, low-density lipoprotein cholesterol, triglycerides, total cholesterol and UAlb were entered in the logistic regression model, and gender, age, RBG, IAA and UAlb were identified as significant risk factors for UTIs $(P<0.05$; Table 2$)$.

\section{Urine cultures}

One hundred and fifty-two patients with symptoms of UTI and 193 patients without the symptoms of UTI had efficient midstream urine culture. Among the193 patients with asymptomatic UTI, $136(70.47 \%)$ were positive in midstream urine culture and diagnosed as ASB, and 67 (44.08\%) patients were positive in midstream urine culture among the 152 patients symptomatic of UTI. There were significant differences in positive rate of urine culture between the two groups $(P<0.0001$; Figure 1).

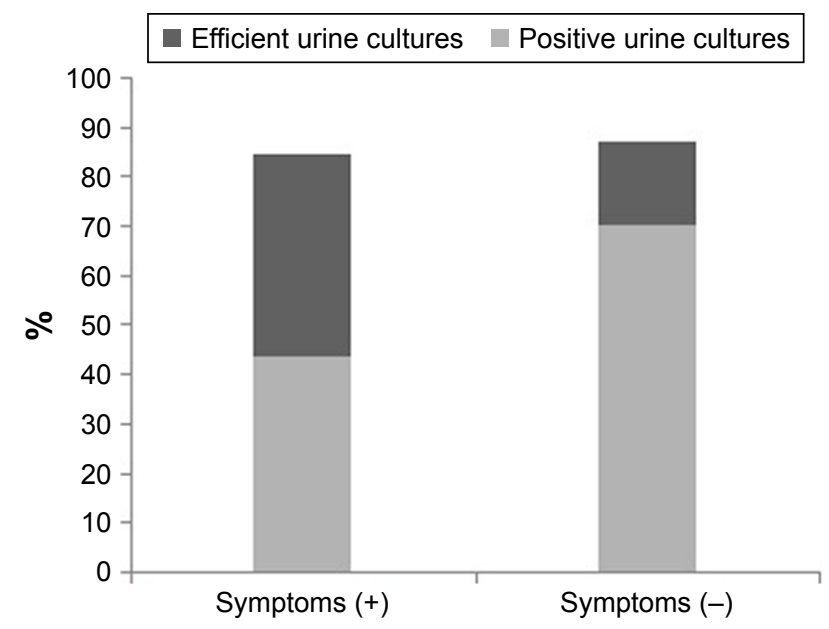

Figure I Urine cultures in diabetic patients.

Notes: Exactly $84.9 \%$ patients with positive UTI symptoms underwent positive urine cultures and $44.08 \%$ of them were positive. Also, $87.33 \%$ patients without UTI symptoms underwent positive urine cultures and $70.47 \%$ were positive. The percentage of positive urine cultures in the two groups was similar; however, the rate of positive urine cultures was significantly higher in symptom (-) group than in symptom $(+)$ group $(P<0.000$ I).

Abbreviation: UTI, urinary tract infection. 
Table 3 The incidence of the uropathogens in diabetic patients

\begin{tabular}{|c|c|c|c|c|c|}
\hline \multirow[t]{2}{*}{ Microorganism } & \multirow{2}{*}{$\begin{array}{l}\text { Number of } \\
\text { patients/ } \\
\text { percentage }\end{array}$} & \multicolumn{2}{|c|}{ Gender } & \multicolumn{2}{|c|}{ Symptoms } \\
\hline & & Male & Female & Positive & Negative \\
\hline Gram-negative & $172 / 84.73 \%$ & 14 & 158 & 56 & 116 \\
\hline Escherichia coli & $134 / 66.01 \%$ & 7 & 127 & 44 & 90 \\
\hline Klebsiella pneumonia & $12 / 5.91 \%$ & 4 & 8 & 3 & 9 \\
\hline Citrobacter freundii & $7 / 3.45 \%$ & 0 & 7 & 2 & 5 \\
\hline Proteus mirabilis & $5 / 2.46 \%$ & 1 & 4 & 2 & 3 \\
\hline Enterobacter sp. & $5 / 2.46 \%$ & 0 & 5 & 3 & 2 \\
\hline Acinetobacter & $4 / 1.97 \%$ & 0 & 4 & I & 3 \\
\hline Pseudomonas sp. & $3 / 1.48 \%$ & 2 & 1 & I & 2 \\
\hline Others* & $2 / 0.99 \%$ & 0 & 2 & 0 & 2 \\
\hline Gram-positive & $24 / 11.82 \%$ & 8 & 16 & 10 & 14 \\
\hline Enterococcus & $12 / 5.91 \%$ & 4 & 8 & 5 & 7 \\
\hline Staphylococcus & $9 / 4.43 \%$ & 4 & 5 & 3 & 6 \\
\hline Streptococcus sp. & $2 / 0.99 \%$ & 0 & 2 & I & I \\
\hline Corynebacterium & $\mathrm{I} / 0.49 \%$ & 0 & I & I & 0 \\
\hline Fungus & $7 / 3.45 \%$ & 4 & 3 & 1 & 6 \\
\hline Candida albicans & $4 / 1.97 \%$ & 3 & I & I & 3 \\
\hline Candida parapsilosis & $2 / 0.99 \%$ & I & I & 0 & 2 \\
\hline Candida tropicalis & $\mathrm{I} / 0.49 \%$ & 0 & I & 0 & I \\
\hline Total & $203 / 100 \%$ & 26 & 177 & 67 & 136 \\
\hline
\end{tabular}

Note: $*$ Others $=$ Morganella morgani and Alcaligenes.

The distribution of the microorganisms that caused UTIs is shown in Table 3. There was no difference between patients with and without UTI symptoms in the distribution of the urine microorganism $(P>0.05)$. Both the patients in ASB group and in the symptom group showed high prevalence of E. coli infections ( $85.29 \%$ and $83.58 \%$, respectively). There were 26 male and 177 female among the patients with positive urine cultures. The positive rate of urine cultures in male UTI patients was lower than in female patients $(38.24 \%$ vs

A

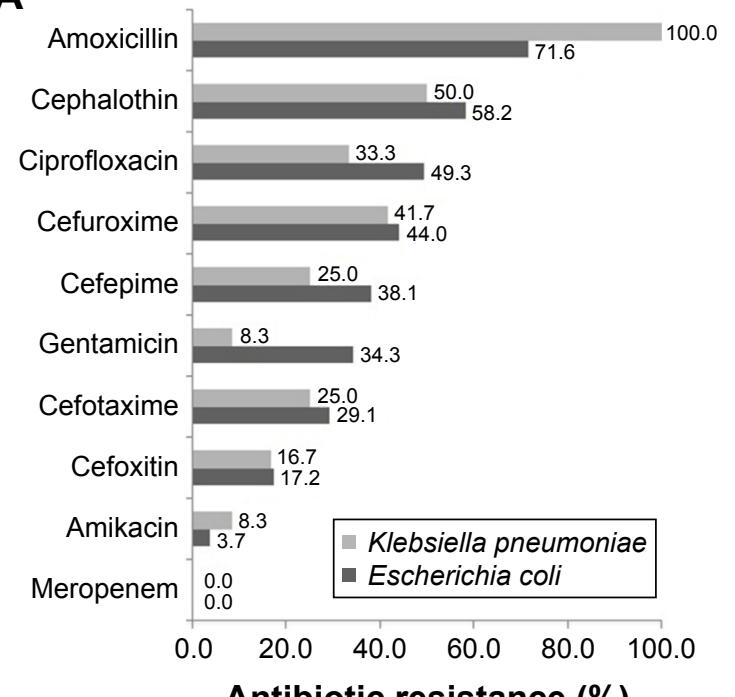

Antibiotic resistance (\%)
$51.91 \%, P<0.05)$. With respect to bacterial infection, male patients had less Gram-negative bacterial infection $(53.85 \%$ vs $89.27 \%, P<0.00001)$ and more Gram-positive bacterial infection $(30.77 \%$ vs $9.04 \%, P<0.01)$ than female patients. Analysis of the pathogenetic microorganisms responsible for infection showed that $E$. coli infection was more common in female patients than in male, while Klebsiella pneumoniae, Enterococcus, and Staphylococcus infections were more common in male patients than in female (all with $P<0.05$; Table 3).

\section{Drug sensitivity test}

The drug resistance pattern of the most popular microorganisms is summarized in Figure 2. E. coli and K. pneumoniae exhibited high resistance to amoxicillin; the resistance rates were $71.6 \%$ and $100 \%$, respectively. However, meropenem was effective for all the microorganisms. Staphylococcus was resistant to penicillin (88.8\%), tetracycline (77.8\%) and erythrocin (77.8\%), but exhibited no resistance to nitrofurantoin and vancomycin. Enterococcus was resistant to tetracycline $(66.7 \%)$ and sensitive to chloramphenicol ( $0 \%$ resistance). The incidences of MDR isolates in E. coli, K. pneumoniae, Staphylococcus and Enterococcus were $50.0 \%, 41.7 \%, 33.3 \%$ and $66.7 \%$, respectively. There was no significant difference in MDR occurrence among these four microorganisms $(P>0.05)$.

\section{Discussion}

In this study, the prevalence of UTIs in diabetic patients was $11.2 \%$, which was similar to that reported in Asia in other

B

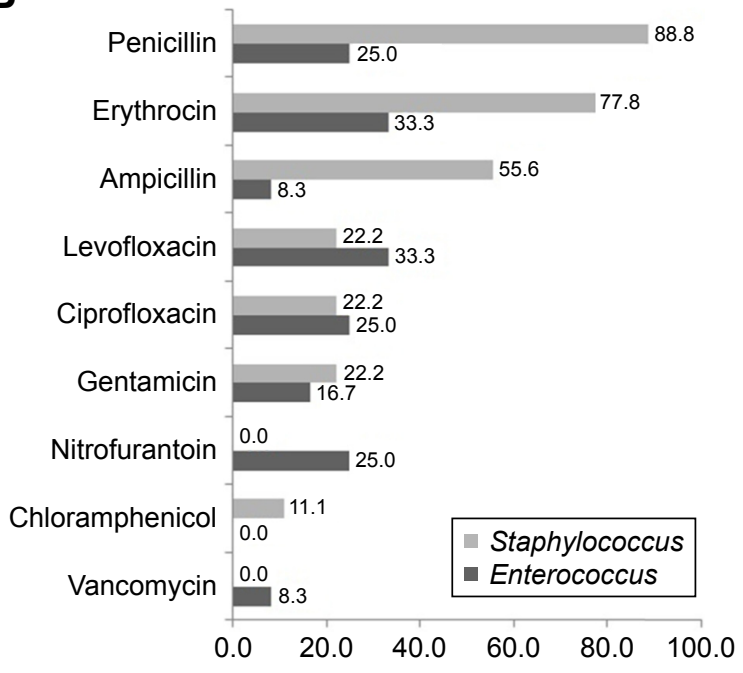

Antibiotic resistance (\%)

Figure 2 The antibiotic resistance to various pathogens in urine culture of diabetic patients.

Notes: (A) Resistance pattern of various commonly used antibiotics in the two most popular Gram-negative organisms. (B) Resistance pattern of various commonly used antibiotics in the two most popular Gram-positive organisms. 
studies (range 13.0\%-24.4\%). ${ }^{17}$ However, the frequency of ASB was lower in our study (only 3.7\%) than previous studies (varied from $6.1 \%$ to $26.6 \%$ ). ${ }^{6,9}$ This might be because both male and female diabetic patients were included in our study, while the previous studies generally included female diabetic patients. In our study, prevalence of UTIs in diabetic women was about 6-fold higher compared with that in diabetic men, which was related to the characteristics of female urinary tract. Beside the female gender, old age and microalbuminuria were also risk factors of UTIs in diabetic patients; however, BMI and duration of diabetes had no relation with UTIs. The results were in accordance with previous studies. ${ }^{6,18,19}$

The reason why diabetic patients tend to get UTIs is not fully understood. HbA1c seems not to be associated with the risk of UTIs, ${ }^{11,18,20}$ while some studies suggested that $\mathrm{HbA} 1 \mathrm{c}$ may be a risk factor of ASB. ${ }^{6,21,22}$ The high levels of $\mathrm{HbA} 1 \mathrm{c}$ in ASB patients may depend on the long duration of infections caused by overlooked illness. ${ }^{23}$ In our study, there was no difference in $\mathrm{HbA1c}$ between UTI patients and non-UTI patients. However, RBG in UTI patients was significantly higher than in non-UTI patients, and RBG was an independent risk factor of UTIs in diabetic patients. The high levels of RBG might be an acute oxidative stress reaction caused by infections. Therefore, the association between RBG and UTIs is stronger than between HbA1c and UTIs.

Although the relationship between diabetes-associated autoantibodies (Islet cell antibody [ISA], IAA, GAD-Ab or protein tyrosine phosphatase antibody IA-2A) and virus infections in type 1 diabetes has been confirmed by several studies, ${ }^{24-26}$ we did not know whether the high levels of IAA and GAD-Ab in UTI patients were related to bacterial infection. To our knowledge, the high levels of IAA and GAD-Ab might imply deterioration of islet cells or decreased blood glucose control in diabetic patients with UTIs.

Unexpectedly, we found that the rate of positive urine cultures was higher in asymptomatic patients than in symptomatic patients, and the incidence rate of septicemia was considerable in the two groups (6 vs 5 patients), which meant the severity of ABS and symptomatic UTIs was similar in diabetic patients. So, we suggest that urine analysis and urine culture should be conducted routinely in diabetes patients.

The most common pathogenic microorganism isolated from UTI patients was E. coli $(66.01 \%)$. K. pneumoniae, Enterococcus and Staphylococcus infections were also common in our study. The results are similar to those of other studies. ${ }^{27-29}$ The incidence of MDR E. coli in our study was $50 \%$, which was similar to the general population in Asia (about 52\%-81\%). ${ }^{30-32}$ In the USA, the incidence of MDR E. coli increased from $9.1 \%$ in 2001 to $17.0 \%$ in $2010 .{ }^{33}$
The different incidences of MDR $E$. coli infections might be due to the racial differences and overuse of antibiotics in some countries.

The most effective antibiotics on Gram-negative bacterial infections were meropenem and amikacin in our study, which is similar to that reported in a study in Bangladesh. ${ }^{28}$ Vancomycin and nitrofurantoin proved to be effective drugs against Gram-positive bacterial infections. Chloramphenicol is a very old drug; however, it was still effective on Staphylococcus infection and no resistant isolate was found.

Enterococcus showed 25\% resistance to nitrofurantoin. Because of the prevalence of extended-spectrum betalactamases produced by bacterial pathogens, ${ }^{34,35}$ amoxicillin showed $100 \%$ resistance to $E$. coli and $71.6 \%$ resistance to $K$. pneumoniae. Furthermore, penicillin and ampicillin also showed high resistance to Gram-positive bacteria. The resistance of $E$. coli to cephalosporins, including cefoxitin, cefotaxime, cefepime, cefuroxime, cephalothin, varied from $17.2 \%$ to $58.2 \%$. These antibiotics were commonly used in our hospital. Although the resistance rates in diabetic patients were similar to non-diabetic patients, ${ }^{27}$ our results suggest cautious use of these antibiotics for the treatment of UTIs.

The limitation of this study is that of the total studied population, only 345 patients underwent positive urine cultures and susceptibility test for antibiotics. In future, we will further expand the sample size of patients with T2D if possible.

In summary, our study suggested that female gender, old age, microalbuminuria, high RBG and IAA were risk factors for UTIs in diabetic patients. The UTIs might affect the islet function or blood glucose control in patients with T2D. The rate of positive urine cultures was higher in asymptomatic patients than in symptomatic patients, and the severity of ABS and symptomatic UTIs was similar in patients with T2D. We suggest that a routine urine analysis or urine culture should be conducted in diabetes patients who have the identified risk factors. E. coli was the most common pathogenic microorganism isolated from diabetic patients with UTIs, and meropenem was the most effective antibiotic on E. coli that was resistant to amoxicillin. When a doctor decides to prescribe antibiotics to a diabetic patient with UTIs, drug sensitivity test should be performed.

\section{Acknowledgment}

We appreciate the support of the nursing and technical staff of Medical Records Room and Nanjing Diabetic Center, Nanjing Hospital Affiliated to Nanjing Medical University.

\section{Disclosure}

The authors report no conflicts of interest in this work. 


\section{References}

1. Shah BR, Hux JE. Quantifying the risk of infectious diseases for people with diabetes. Diabetes Care. 2003;26(2):510-513.

2. Muller LM, Gorter KJ, Hak E, et al. Increased risk of common infections in patients with type 1 and type 2 diabetes mellitus. Clin Infect Dis. 2005;41(3):281-288.

3. Gallacher SJ, Thomson G, Fraser WD, Fisher BM, Gemmell CG, MacCuish AC. Neutrophil bactericidal function in diabetes mellitus: evidence for association with blood glucose control. Diabet Med. 1995; 12(10):916-920.

4. Delamaire M, Maugendre D, Moreno M, Le Goff MC, Allannic H, Genetet B. Impaired leucocyte functions in diabetic patients. Diabet Med. 1997;14:29-34.

5. Fu AZ, Iglay K, Qiu Y, Engel S, Shankar R, Brodovicz K. Risk characterization for urinary tract infections in subjects with newly diagnosed type 2 diabetes. J Diabetes Complications. 2014;28(6):806-810.

6. Turan H, Serefhanoglu K, Torun AN, et al. Frequency, risk factors, and responsible pathogenic microorganisms of asymptomatic bacteriuria in patients with type 2 diabetes mellitus. Jpn J Infect Dis. 2008;61(3): 236-238.

7. Tourret J, Bagnis CI, Denamur E. Urinary tract infections in diabetic patients. Rev Prat. 2014;64:980-983.

8. Zhanel GG, Harding GK, Guay DR. Asymptomatic bacteriuria. Which patients should be treated? Arch Intern Med. 1990;150(7):1389-1396.

9. Boroumand MA, Sam L, Abbasi SH, Salarifar M, Kassaian E, Forghani S. Asymptomatic bacteriuria in type 2 Iranian diabetic women: a cross sectional study. BMC Womens Health. 2006;6:4.

10. Nicolle LE. A practical guide to antimicrobial management of complicated urinary tract infection. Drugs Aging. 2001;18(4):243-254.

11. Nicolle LE. Asymptomatic bacteriuria in diabetic women. Diabetes Care. 2000;23:722-723.

12. Sumardi R, Mochtar CA, Junizaf, et al. Prevalence of urinary incontinence, risk factors and its impact: multivariate analysis from Indonesian nationwide survey. Acta Med Indones. 2014;46(3):175-182.

13. Bonadio M, Meini M, Spitaleri P, Gigli C. Current microbiological and clinical aspects of urinary tract infections. Eur Urol. 2001;40:439-444.

14. Kahlmeter G, Poulsen HO. Antimicrobial susceptibility of Escherichia coli from community-acquired urinary tract infections in Europe: the ECO·SENS study revisited. Int J Antimicrob Agents. 2012;39:45-51.

15. Kuzdan C, Soysal A, Culha G, Altinkanat G, Soyletir G, Bakir M. Three-year study of health care-associated infections in a Turkish pediatric ward. J Infect Dev Ctries. 2014;8:1415-1420.

16. Gupta S, Kapur S, Padmavathi D. Comparative prevalence of antimicrobial resistance in community-acquired urinary tract infection cases from representative States of northern and southern India. J Clin Diagn Res. 2014;8(9):DC09-12.

17. Irwin DE, Kopp ZS, Agatep B, Milsom I, Abrams P. Worldwide prevalence estimates of lower urinary tract symptoms, overactive bladder, urinary incontinence and bladder outlet obstruction. BJU Int. 2011;108(7):1132-1138.

18. Ishay A, Lavi I, Luboshitzky R. Prevalence and risk factors for asymptomatic bacteriuria in women with Type 2 diabetes mellitus. Diabet Med. 2006;23(2):185-188.

19. Papazafiropoulou A, Daniil I, Sotiropoulos A, et al. Prevalence of asymptomatic bacteriuria in type 2 diabetic subjects with and without microalbuminuria. BMC Res Notes. 2010;3:169.

Therapeutics and Clinical Risk Management

\section{Publish your work in this journal}

Therapeutics and Clinical Risk Management is an international, peerreviewed journal of clinical therapeutics and risk management, focusing on concise rapid reporting of clinical studies in all therapeutic areas, outcomes, safety, and programs for the effective, safe, and sustained use of medicines. This journal is indexed on PubMed Central, CAS,
20. Boyko EJ, Fihn SD, Scholes D, Abraham L, Monsey B. Risk of urinary tract infection and asymptomatic bacteriuria among diabetic and nondiabetic postmenopausal women. Am J Epidemiol. 2005;161(6): 557-564.

21. Bonadio M, Boldrini E, Forotti G, et al. Asymptomatic bacteriuria in women with diabetes: influence of metabolic control. Clin Infect Dis. 2004;38(6):e41-e45.

22. Keleştimur F, Unal A, Paşaoğlu H, Başar E, Kiliç H, Doğanay M. [Asymptomatic bacteriuria in patients with diabetes mellitus]. Mikrobiyol Bul. 1990;24(2):126-132. Turkish.

23. Dalal S, Nicolle L, Marrs CF, Zhang L, Harding G, Foxman B. Longterm Escherichia coli asymptomatic bacteriuria among women with diabetes mellitus. Clin Infect Dis. 2009;49(4):491-497.

24. Lempainen J, Tauriainen S, Vaarala $\mathrm{O}$, et al. Interaction of enterovirus infection and cow's milk-based formula nutrition in type 1 diabetesassociated autoimmunity. Diabetes Metab Res Rev. 2012;28(2):177-185.

25. Betterle C, Fabris P, Zanchetta R, et al. Autoimmunity against pancreatic islets and other tissues before and after interferon-alpha therapy in patients with hepatitis C virus chronic infection. Diabetes Care. 2000; 23(8):1177-1181.

26. Blomqvist $M$, Juhela $\mathrm{S}$, Erkkila $\mathrm{S}$, et al. Rotavirus infections and development of diabetes-associated autoantibodies during the first 2 years of life. Clin Exp Immunol. 2002;128(3):511-515.

27. Bonadio M, Costarelli S, Morelli G, Tartaglia T. The influence of diabetes mellitus on the spectrum of uropathogens and the antimicrobial resistance in elderly adult patients with urinary tract infection. $B M C$ Infect Dis. 2006;6:54.

28. Shill MC, Huda NH, Moain FB, Karmakar UK. Prevalence of uropathogens in diabetic patients and their corresponding resistance pattern: results of a survey conducted at diagnostic centers in Dhaka, Bangladesh. Oman Med J. 2010;25(4):282-285.

29. Ronald A, Ludwig E. Urinary tract infections in adults with diabetes. Int J Antimicrob Agents. 2001;17(4):287-292.

30. Wang Y, Zhao S, Han L, et al. Drug resistance and virulence of uropathogenic Escherichia coli from Shanghai, China. J Antibiot (Tokyo). 2014;67(12):799-805.

31. Sabir S, Ahmad Anjum A, Ijaz T, Asad Ali M, Ur Rehman Khan M, Nawaz M. Isolation and antibiotic susceptibility of $E$. coli from urinary tract infections in a tertiary care hospital. Pak J Med Sci. 2014;30(2): 389-392.

32. Hasan AS, Nair D, Kaur J, Baweja G, Deb M, Aggarwal P. Resistance patterns of urinary isolates in a tertiary Indian hospital. J Ayub Med Coll Abbottabad. 2007;19(1):39-41.

33. Sanchez GV, Baird AM, Karlowsky JA, Master RN, Bordon JM. Nitrofurantoin retains antimicrobial activity against multidrug-resistant urinary Escherichia coli from US outpatients. J Antimicrob Chemother. 2014;69(12):3259-3262.

34. Lu PL, Liu YC, Toh HS, et al. Epidemiology and antimicrobial susceptibility profiles of Gram-negative bacteria causing urinary tract infections in the Asia-Pacific region: 2009-2010 results from the Study for Monitoring Antimicrobial Resistance Trends (SMART). Int $J$ Antimicrob Agents. 2012;40(Supp1):S37-S43.

35. Meier S, Weber R, Zbinden R, Ruef C, Hasse B. Extended-spectrum $\beta$-lactamase-producing Gram-negative pathogens in communityacquired urinary tract infections: an increasing challenge for antimicrobial therapy. Infection. 2011;39(4):333-340.

\section{Dovepress}

EMBase, Scopus and the Elsevier Bibliographic databases. The manuscript management system is completely online and includes a very quick and fair peer-review system, which is all easy to use. Visit $\mathrm{http}: / / \mathrm{www}$.dovepress.com/testimonials.php to read real quotes from published authors. 